\title{
Let-7i inhibits the malignant phenotype of osteosarcoma cells by targeting Aurora-B
}

\author{
GUO MEI ZHANG ${ }^{1 *}$, XIN HUA LONG ${ }^{2 *}$, JIA MING LIU ${ }^{2 *}$, LIANG BO ZHU ${ }^{2}$, \\ XUAN YIN CHEN ${ }^{2}$, SHAN HU HUANG ${ }^{2}$, ZHI HONG ZHANG ${ }^{2}$ and ZHI LI LIU ${ }^{2}$ \\ ${ }^{1}$ Department of Orthopedics, The Second Affiliated Hospital of Nanchang University; ${ }^{2}$ Department of Orthopedics, \\ The First Affiliated Hospital of Nanchang University, Nanchang, Jiangxi 330006, P.R. China
}

Received May 14, 2014; Accepted April 10, 2015

DOI: $10.3892 / \mathrm{mmr} .2015 .3798$

\begin{abstract}
Our previous study indicated that Aurora-B is involved in osteosarcoma (OS) cell invasion and metastasis; however, the mechanism underlying Aurora-B overexpression in OS remains unknown. In the present study, significantly downregulated let-7i expression in OS tissues and OS cells was observed compared with that in normal adjacent tumorous tissues and human osteoblast cell lines. Bioinformatic predictions have revealed a conserved binding site in a microRNA locus on Aurora-B, suggesting the potential of let-7i targeting the Aurora-B gene. To validate this, a luciferase reporter assay was performed on OS cells. The results indicated that Aurora-B is a likely to be a direct target negatively regulated by let-7i. The expression of let-7i in OS cells was restored by infection with let-7i mimics. Results revealed that Aurora-B mRNA and protein expression levels were significantly decreased. Furthermore, the proliferation, migration and invasion abilities of OS cells were significantly suppressed by infection with let-7i mimics. Notably, the inhibitory effect of silencing Aurora-B by LV-shAurora-B on cell proliferation, migratory and invasive ability was significantly lower than that by let-7i mimics, which indicated that let-7i inhibits cell malignant phenotypes partially by targeting Aurora-B in OS cells. All data suggested that let-7i may be a novel potential target for OS treatment.
\end{abstract}

\section{Introduction}

Osteosarcoma (OS) is the most common type of primary malignant tumor of the bone in young adolescents and infants (1). Pulmonary metastasis is the predominant cause of

Correspondence to: Mr. Zhi Li Liu or Mr. Zhi Hong Zhang, Department of Orthopedics, The First Affiliated Hospital of Nanchang University, 17 Yong Wai Zheng, Nanchang, Jiangxi 330006, P.R. China

E-mail: zgm7977@163.com

E-mail: 13803505665@163.com

*Contributed equally

Key words: let-7i, Aurora-B, osteosarcoma, migration, invasion mortality in patients with OS. Studies have demonstrated that the five-year survival rate of patients with metastatic diseases was $<20 \%$ (1-3). Clearly, a comprehensive understanding of the biological mechanisms of this malignancy is required for the management of OS.

Accumulating studies have demonstrated that miRNAs are critical in cell proliferation, apoptosis and metastasis (4-6). Let-7 is one of the most extensively investigated miRNAs in the Caenorhabditis elegans genome. It regulated seam cell terminal differentiation, possibly by acting as a regulator of multiple genes required for cell cycle and proliferation. Eleven members of the let-7 cluster have been identified in the human genome. Notably, the let-7 family is one of the first reported tumor suppressor miRNAs in cancer, which negatively regulates RAS and is expressed at lower levels in lung tumors than in normal lung tissue (7). The let-7 cluster has been shown to be significantly correlated with the occurrence and development of cancer, suggesting that it is involved in the regulation of oncogenic pathways in numerous types of tumors $(8,9)$. Reduced expression of let-7 has also been associated with shortened postoperative survival in patients with lung cancer (8). In addition, enhanced expression of let-7 family members is able to inhibit malignant tumor cell growth $(9,10)$. Let-7i is one member of the let-7 family. Recently, studies have indicated that let-7i is involved in cancer metastasis (11). However, it is unknown whether let-7i is essential in OS development and metastasis.

In the present study, the association between let-7i and Aurora-B in OS tissue and cell lines were investigated, in order to elucidate the possible molecular mechanisms of metastasis of OS and improve the therapeutic strategies for the management of OS.

\section{Materials and methods}

Patients and clinical samples. Twenty-one OS specimens were collected prior to neoadjuvant chemotherapy in the Department of Orthopedics, The First Affiliated Hospital of Nanchang University and The Cancer Hospital of Jiangxi Province (Nanchang, China) between 2009 and 2012. The matched normal tissues obtained from an area $5 \mathrm{~cm}$ from the tumor margin, were used as negative controls. The diagnosis was confirmed by two pathologists. The study protocol and operational procedures were approved by the Human Ethics Committee of Nanchang 
University, and a signed informed consent form was obtained from all patients or patients' family members.

Cell lines and cell culture. The U2-OS and HOS human osteosarcoma cell lines and the human osteoblast cell line HOB was obtained from American Type Culture Collection (Manassas, VA, USA), and routinely cultured in Dulbecco's modified Eagle's medium (Hyclone, Logan, UT, USA) supplemented with 10\% fetal bovine serum (FBS; Sigma Aldrich, St. Louis, $\mathrm{MO}, \mathrm{USA}$ ) in a humidified $37^{\circ} \mathrm{C}$ incubator containing $5 \% \mathrm{CO}_{2}$.

$R N A$ isolation and reverse transcription-quantitative polymerase chain reaction ( $R T-q P C R)$. The formalin-fixed paraffin-embedded (FFPE) OS tissues and adjacent tumorous tissues were obtained for microRNA isolation using the Qiagen RN easy FFPE protocol (Qiagen, Valencia, CA, USA) according to Kelly et al (12). The let-7i expression levels were evaluated by RT-qPCR (StepOne ${ }^{\mathrm{TM}}$; Bio-Rad Laboratories, Inc., Hercules, CA, USA), using U6 snRNA as the endogenous reference gene. Total RNA from OS cells was extracted using $72 \mathrm{~h}$ culture with TRIzol (Invitrogen Life Technologies, Carlsbad, CA, USA). Reverse transcription was performed with $2 \mathrm{mg}$ total RNA using PrimeScript RT Reagent Kit (Takara Bio, Inc., Otsu, Japan). Then each sample was analyzed by qPCR under the conditions described in the manufacturer's instructions for SYBR Premix Ex Tap II (Invitrogen Life Technologies): $50^{\circ} \mathrm{C}$ for $2 \mathrm{~min}, 95^{\circ} \mathrm{C}$ for $2 \mathrm{~min}$, followed by 40 cycles of $95^{\circ} \mathrm{C}$ for $15 \mathrm{sec}$ and $60^{\circ} \mathrm{C}$ for $30 \mathrm{sec}$. Relative expression was calculated using the $2-\Delta \Delta \mathrm{Ct}$ method. All procedures were conducted according to the manufacturer's instructions. The primer sequences can be found in Table I.

Cell growth assay. OS cells were cultured into five 96-well tissue culture plates at a cell density of 5,000 cells $/ \mathrm{ml}$ in a volume of $200 \mu \mathrm{l}$ culture medium. A total of $20 \mu \mathrm{l}$ 3-(4,5-Dimethylthiazol-2-yl)-2,5-diphenyltetrazolium bromide (MTT; $0.5 \mathrm{mg} / \mathrm{ml}$; Beyotime Institute of Biotechnology, Beijing, China) was added to each well, and the wells were incubated at $37^{\circ} \mathrm{C}$ for $4 \mathrm{~h}$. Subsequently, the supernatant was removed, and $20 \mu 1$ dimethyl sulfoxide was added to each well to dissolve the formazan crystals at $37^{\circ} \mathrm{C}$ for $20 \mathrm{~min}$. The optical density values were measured at $490 \mathrm{~nm}$ wave length in triplicate using Synergy ${ }^{\mathrm{TM}}$ HT (BioTek Instruments, Inc., Winooski, VT, USA).

Western blot analysis. Total protein from cells was extracted using radioimmunoprecipitation lysis buffer (Beyotime Institute of Biotechnology) containing $60 \mu \mathrm{g} / \mathrm{ml}$ PMSF (Solarbio, Beijing, China). Protein concentration was determined by a Bradford assay (Bio-Rad Laboratories, Inc.). Western blot analysis was conducted using antibodies against Aurora-B (EP1009Y; Abcam, Cambridge, UK) and $\beta$-actin (sc-130657; Santa Cruz Biotechnology Inc., Santa Cruz, CA, USA). The immune complexes were detected with a pro-light horseradish peroxidase kit (Life Technologies, Carlsbad, CA, USA). Six independent experiments were performed over multiple days.

Migration assay. Cell migration was assessed by a wound healing assay, which determined the ability of the cells to move into a cellular space in two-dimension in vitro. In brief, cells

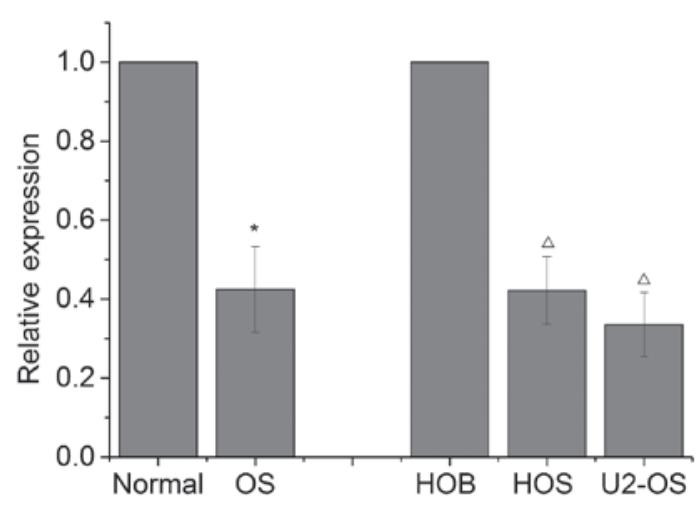

Figure 1. Expression level of let-7i in OS tissues and cell lines was evaluated by reverse transcription-quantitative polymerase chain reaction. The results revealed that expression of let-7i was significantly downregulated in OS tissues and OS cells compared with that in normal tissues and osteoblast cells, suggesting that the let-7i may be essential in OS. (Columns, mean $(n=6)$; bars, standard deviation. ${ }^{*} \mathrm{P}<0.05$ vs. normal adjacent tumorous tissues, ${ }^{\Delta} \mathrm{P}<0.05$ vs. osteoblast cell lines). OS, osteosarcoma.

were grown to $100 \%$ confluence in 6-well tissue culture plastic dishes to a density of $\sim 5 \times 10^{6}$ cells/well. The cells were denuded by dragging a rubber policeman (Fisher Scientific, Hampton, $\mathrm{NH}, \mathrm{USA}$ ) through the center of the plate. Cultures were rinsed with phosphate-buffered saline and replaced with fresh quiescent medium alone or containing $10 \%$ FBS, following which the cells were incubated at $37^{\circ} \mathrm{C}$ for $24 \mathrm{~h}$. Images were captured at 0 and $24 \mathrm{~h}$ using an ECLIPSE-TS-100 microscope (magnification, x200; Nikon, Tokyo, Japan), and the migrated distance was measured by Image J, version 1.48 (National Center for Biotechnology Information, Bethesda, MD, USA). The cell migration rate was obtained by counting three fields per area and are represented as the average of six independent experiments conducted over multiple days.

Transwell invasion assays. Invasion of OS cells was measured using the BD BioCoatTM BD Matrigel ${ }^{\mathrm{TM}}$ Invasion Chamber (BD Biosciences, Franklin Lakes, NJ, USA) according to the manufacturer's instructions. The medium in the lower chamber contained 5\% fetal calf serum as a source of chemoattractants. Cells were suspended in serum-free medium and added to the upper chambers at the same time. Cells that passed through the Matrigel-coated membrane were stained with Diff-Quik (Sysmex, Kobe, Japan) and photographed (magnification, $\mathrm{x} 400$ ). Images were captured at $24 \mathrm{~h}$, and cell counting was measured by Image $\mathbf{J}$ software. The values for invasion were obtained by counting three fields per membrane and represented as the average of six independent experiments conducted over multiple days.

Lentivirus-vector construction and cell transfection. To construct vectors for downregulating Aurora-B, the sequences of interfering microRNA targeting Aurora-B (5'-CCG GCTCCAAACTGCTCAGGCATAACTCGAGTTATGCCTGA GCAGTTTGGAGTTTTTG-3') were inserted into lentivirus vector GV115 (GeneChem Co., Ltd., Shanghai, China). U2-OS and HOS cells were transfected with lentivirus vectors (GeneChem Co., Ltd.) of downregulating Aurora-B (LV-shAurora-B) and negative lentivirus vectors (LV-Neg), respectively $(\mathrm{MOI}=20)$. The let-7 mimics and negative mimics 
Table I. Primer sequences.

Primer

let-7i-RT

Let-7i-Q-F

U6-RT

U6-Q-F

Q-miR-5p-R

Aurora-B-196 bp-F

Aurora-B-196 bp-R

$\beta$-actin-295 bp-F

$\beta$-actin-295 bp-R
Sequences ( $5^{\prime}$ to $\left.3^{\prime}\right)$

GTCGTATCCAGTGCAGGGTCCG

AGGTATTCGCACTGGAACAGCA

GCGTGAGGTAGTAGTTTG

GTCGTATCCAGTGCAGGGTCCGAGG

TATTCGCACTGGATACGACAAAAAT

GCACTGGACTTGGAGTCA

CAGTGCAGGGTCCGAGGT

AAGGAGAACTCCTACCCCTGG

TTAAGATGTCGGGTGTCCCAC

TCACCCACACTGTGCCATCATCGA

CAGCGGAACCGCTCATTGCCAATGG

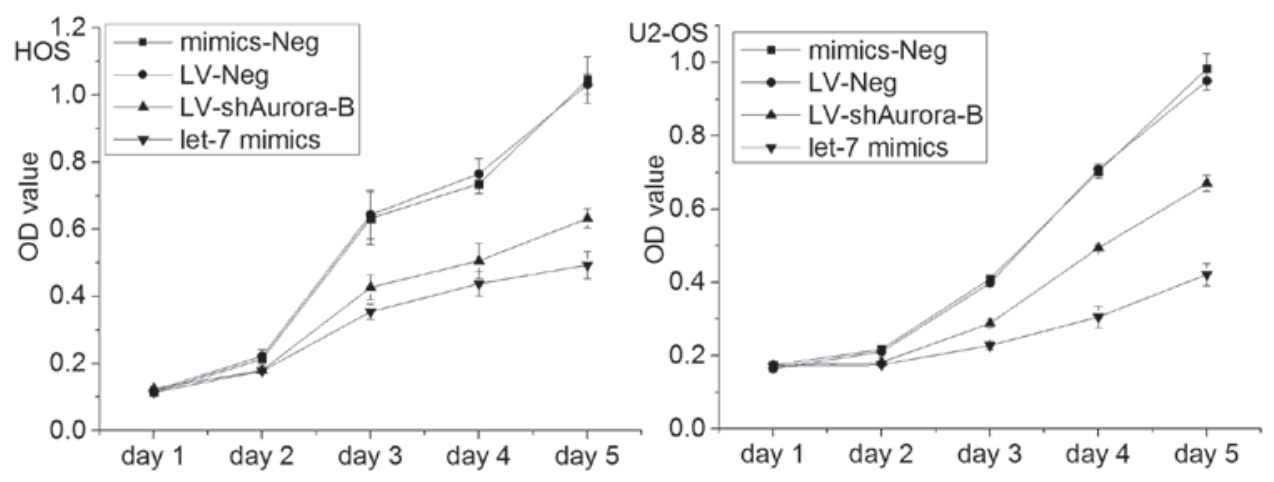

Figure 2. OS cell proliferation was evaluated by an 3-(4,5-dimethylthiazol-2-yl)-2,5-diphenyltetrazolium bromide assay. The results revealed that the viability of OS cells was inhibited by restoration expression of let-7i in OS cells. The inhibitory effect of silencing Aurora-B by LV-shAurora-B on cell proliferation was significantly lower than that by let-7i mimic, which indicated that let-7i inhibits cell proliferation partially by targeting Aurora-B in OS cells. OS, osteosarcoma.

(mimics-Neg) were transfected with Lipofectamine 2000 (Life Technologies). The transfection efficiency was evaluated under the fluorescence microscope (BX61; Olympus, Tokyo, Japan).

Target prediction. Prediction of the Aurora-B 3'-UTR as a miRNA binding target was determined using TargetScan (www. targetscan.org), microRNA (www.microrna.org), and PicTar (pictar.mdc-berlin.de). MiRNAs that were simultaneously predicted by all 3 programs were selected for the present study.

Vector construction and luciferase reporter assay. To generate a luciferase reporter construct, 3'UTR and mutant 3'UTR of Aurora-B were inserted downstream of firefly luciferase in pGL3 (Promega Corporation, Madison, WI, USA). Cells were cotransfected with let-7i and 3'UTR or mutant 3'UTR luciferase reporters, using pRL-TK (Promega Corporation) as the control vector. Luciferase activity was measured using the Dual-Luciferase Assay kit (Promega Corporation) with a beta-counter luminometer (Promega Corporation). Relative luciferase activity was calculated as ratio of the raw firefly luciferase activity to the renilla luciferase activity.

Statistical analysis. All data are presented as the mean \pm standard deviation, an independent-samples t-test was performed for statistical analysis, and $\mathrm{P}<0.05$ was considered to indicate a statistically significant difference. All analyses were performed using SPSS Version 13.0 (SPSS Inc., Chicago, IL, USA).

\section{Results}

Let-7i is downregulated in OS tissues and cell lines. In order to investigate the correlation between let-7i dysregulation and OS, RT-qPCR was conducted to measure let-7i expression in 21 human OS and normal tissues (adjacent tumorous tissues), U2-OS and HOS cell lines and osteoblast cell lines. The results revealed that expression of let-7i was significantly downregulated in OS tissues compared with that in normal tissues (Fig. 1). Furthermore, a decreased expression of let-7i was also observed in OS cells when compared with that in osteoblast cells (Fig. 1). These results suggest that the let-7i may be essential in OS.

Enhanced let-7i expression suppresses cell viability of OS cells in vitro. In order to investigate the involvement of let-7i in the OS malignant phenotype, the expression of let-7i in U2-OS and HOS cells was restored by infection with let-7i mimic. Cell viability was investigated through evaluation of proliferation by MTT assays. The results revealed that the viability of OS cells was inhibited by restoration of expression of let-7i in OS 
A

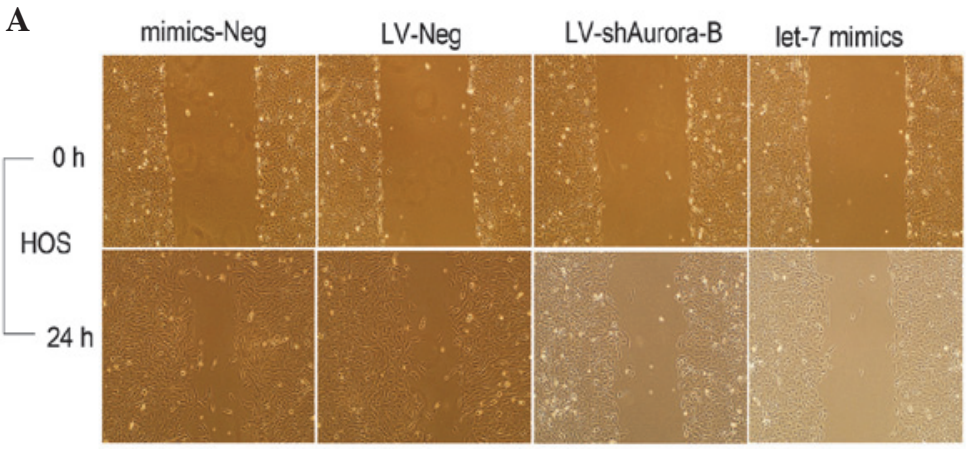

B
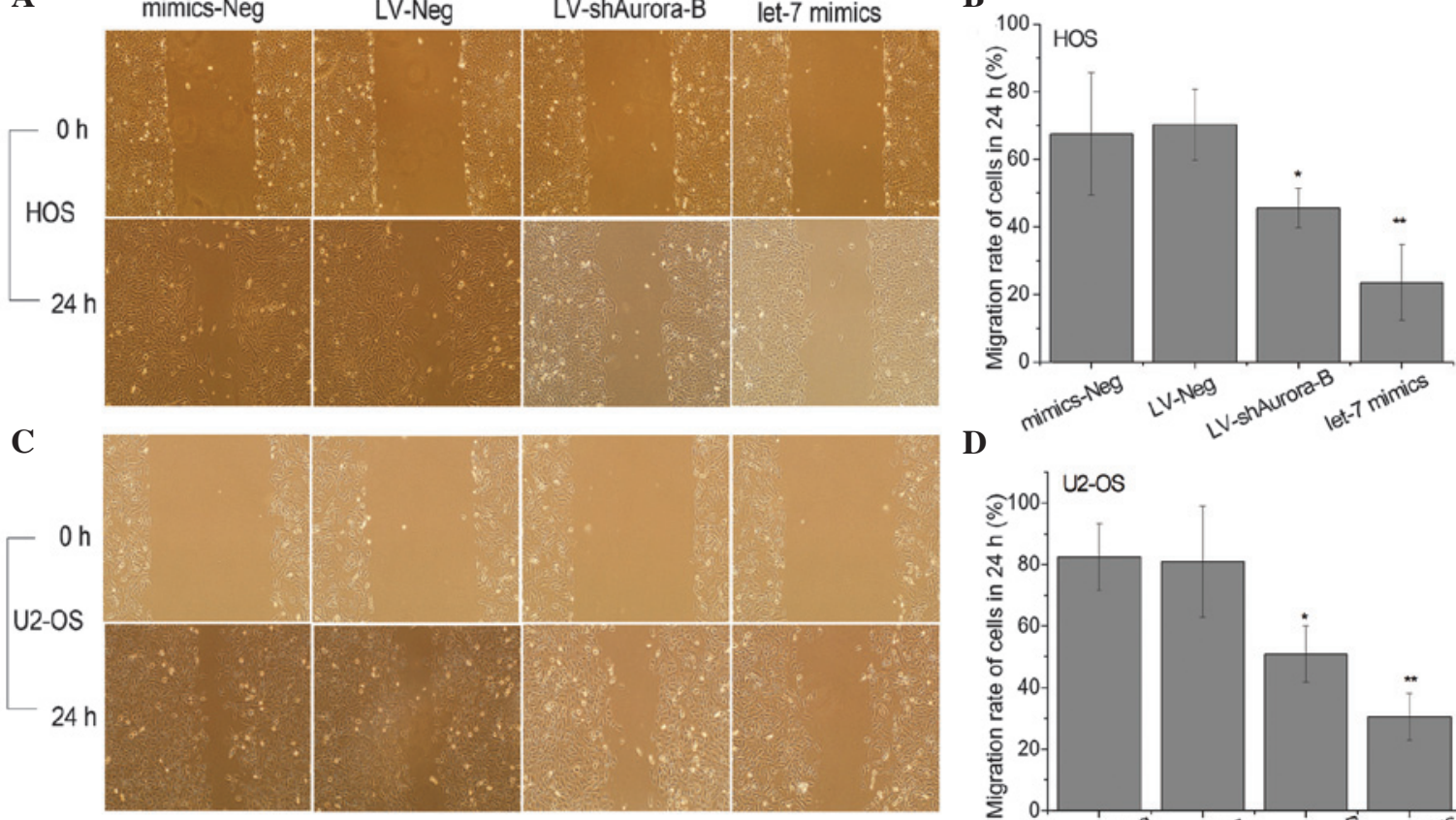

D
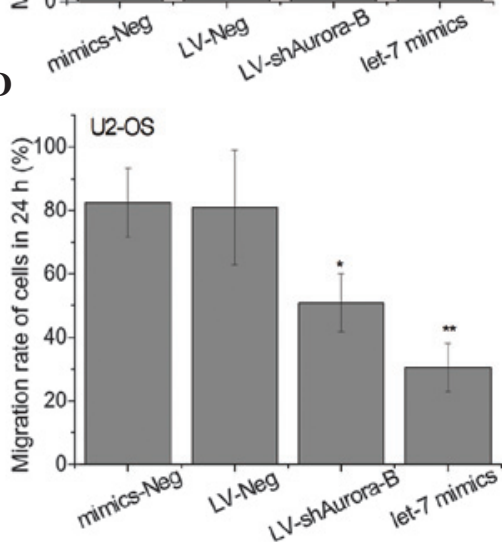

Figure 3. Migratory ability of OS cells was measured by wound healing assays. (A) Representative image of the wound healing assay in the HOS group. (B) The OS cell migratory rate during the wound healing assay in the HOS group. (C) Representative image of the wound healing assay in the U2-OS group. (D) The OS cell migratory rate during the wound healing assay in the U2-OS group. The migratory rate was significantly lower in cells infected with let-7i mimics than that in cells infected with negative mimics, suggesting that enhanced expression of let-7i could inhibit OS cell migration in vitro. The inhibitory effect of silencing Aurora-B by LV-shAurora-B on cell migration was significantly lower than that by let-7i mimic, indicating that let-7i inhibits cell migration partially targeting Aurora-B in OS cells. OS, osteosarcoma.

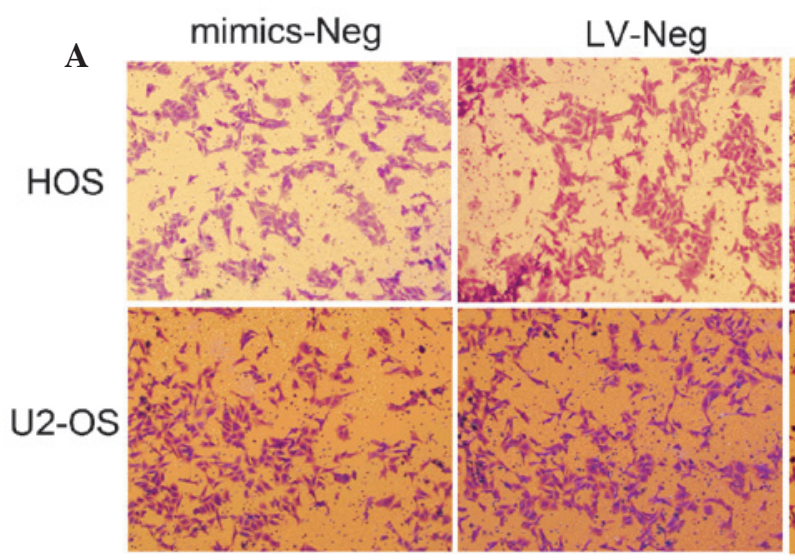

LV-shAurora-B

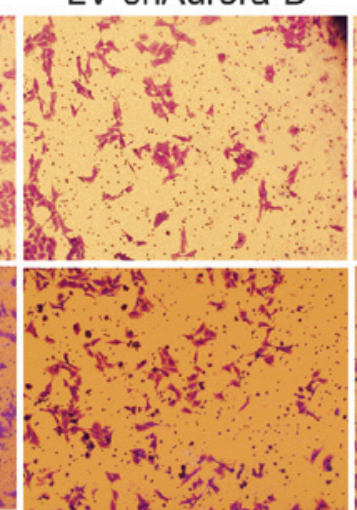

B

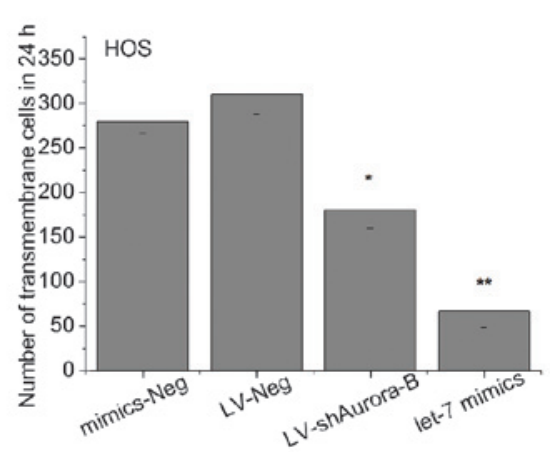

C

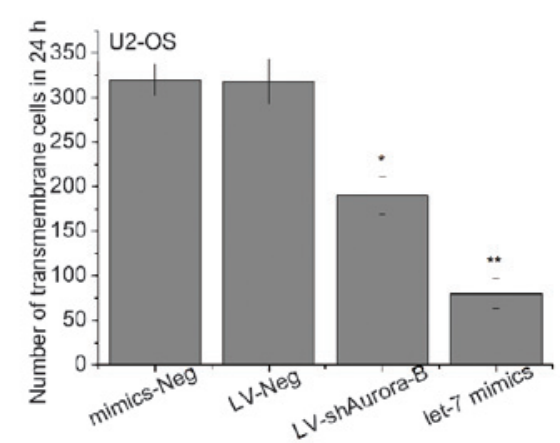

Figure 4. Invasive ability of cells was measured by Transwell assays. (A) Cellular invasion through Matrigel-coated transwells is presented for each group. $(B$ and $C)$ The number of OS cells invading through the membrane in each group. The number of invasive cells was significantly lower in cells infected with let-7i mimics than that in cells infected with negative mimics, suggesting that enhanced expression of let-7i could inhibit OS cell invasion in vitro. The inhibitory effect of silencing Aurora-B by LV-shAurora-B on cell invasive ability was significantly lower than that by let-7i mimics. The results indicated that let-7i inhibits cell invasion partially by targeting Aurora-B in OS cells. 
A

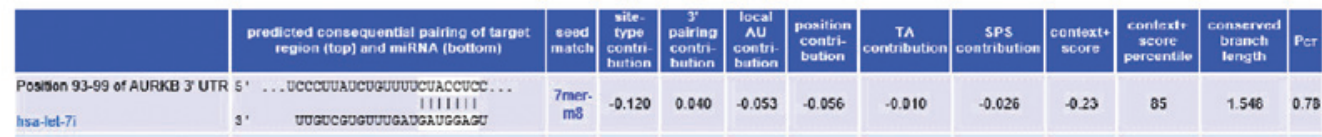

B

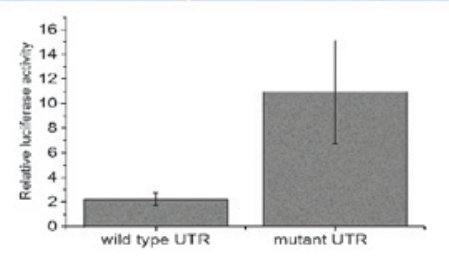

D
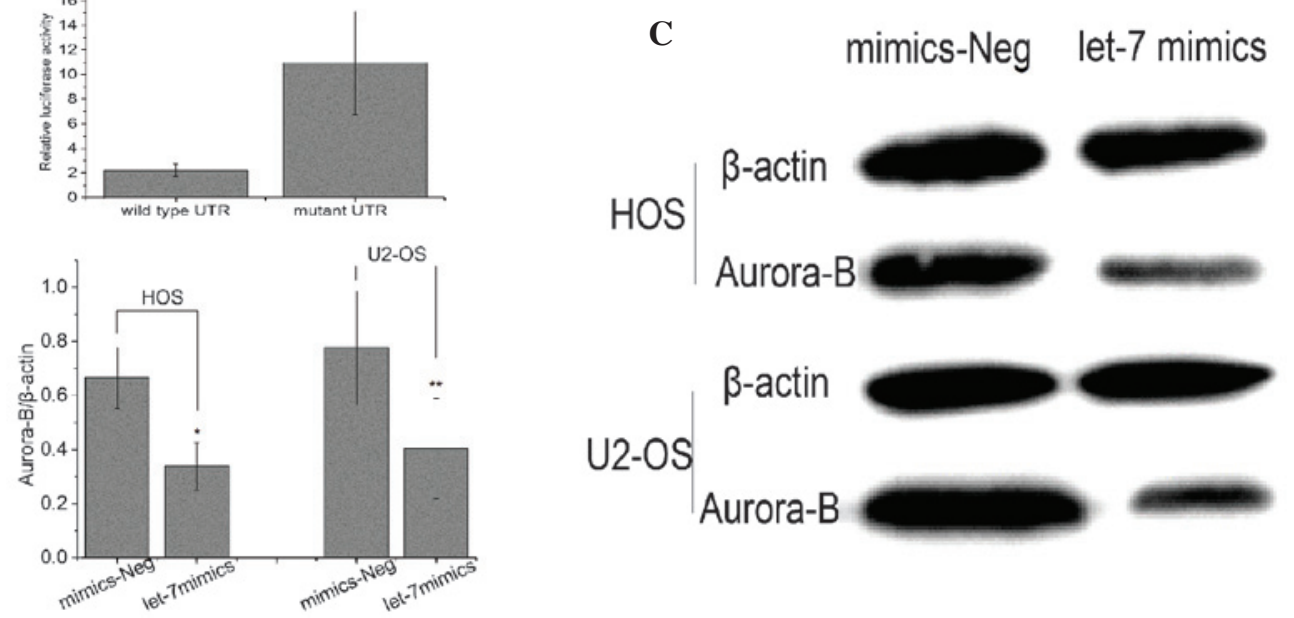

Figure 5. Let-7i negatively regulates Aurora-B expression in OS cells. (A) Aurora-B may be a target gene of let-7i. (B) A luciferase assay suggested that Aurora-B may be targeted by let-7i in OS cells. Aurora-B (C) protein and (D) mRNA were significantly reduced by let-7i mimics in OS cells, suggesting let-7i can negatively regulate Aurora-B expression in OS cells.

cells (Fig. 2). These data indicated that let-7i decreased OS cell viability in vitro.

Enhanced let-7i expression suppresses cell migration and invasion of OS cells in vitro. For investigating the effect of let-7i on migration and invasion of OS cells, the let-7i mimic was used to restore let-7i expression in U2-OS and HOS cells, and the migratory and invasive ability of cells were measured by wound healing and Transwell assays. The migratory rate and number of invasive cells were significantly lower in cells infected with let-7i mimics than that in cells infected with negative mimics (Figs. 3 and 4). These results suggest that enhanced expression of let-7i inhibited OS cell migration and invasion in vitro.

Let-7i negatively regulates Aurora-B expression in OS cells. Our previous study indicated that Aurora-B is involved in OS cell invasion and metastasis (13), and recent studies have demonstrated that Aurora-B is a target of let-7a (14). Therefore, in order to explore whether Aurora-B is a target of let-7i, the prediction was performed by two target prediction websites [Pictar and Targetscan (15)]. It was found that Aurora-B may be the target gene of let-7i (Fig. 5A). In addition, to investigate whether Aurora-B was regulated by let-7i through direct binding to its $3^{\prime}$ untranslated region (UTR), the full-length wild-type and mutant fragments of Aurora-B mRNA 3'-UTR were constructed, and inserted into the region immediately downstream of a luciferase reporter gene. Subsequently, let-7i mimic oligos were co-transfected with different luciferase 3'-UTR constructs into U2-OS cells. Results revealed that let-7i decreased the relative luciferase activity in the wild-type 3'-UTR of Aurora-B. Furthermore, luciferase activity was not significantly decreased in the UTRs with mutant binding sites compared with the mut-type counterparts (Fig. 5B). The results suggested that Aurora-B may be targeted by let-7i in OS cell.

To further determine let-7i negative regulation of Aurora-B expression in OS cells, the let-7i expression in U2-OS and HOS cells was restored by infection with let-7i mimic, and Aurora-B mRNA and protein expression was analyzed by RT-qPCR and western blot analysis. The results show that expression levels of Aurora-B protein and mRNA were significantly lower in cells infected with let-7i mimic than that in cells infected with negative mimic, suggesting that let-7i can negatively regulate Aurora-B expression in OS cells (Fig. 5C and D).

Let-7i inhibits OS cell malignant phenotype partly by targeting Aurora- $B$. To explore the functional correlation between let-7i and Aurora-B in OS. The U2-OS and HOS cells were infected with let-7i mimic and LV-shAurora-B, respectively. It was found that the level of Aurora-B protein expression significantly decreased in cells infected with LV-shAurora-B or let-7i mimic. Furthermore, the effect of enhancing let-7i and silencing Aurora-B on cell proliferation, migration and invasion was investigated. Results revealed that the inhibitory effect of silencing Aurora-B by LV-shAurora-B on cell proliferation, migratory and invasive ability was significantly lower than that by let-7i mimic (Figs. 2-4). These results indicated that let-7i inhibits the malignant phenotype partially by targeting Aurora-B in OS cells.

\section{Discussion}

In the present study, it was demonstrated that let-7i expression in OS tissues and cell lines was downregulated compared with that in normal tissues and osteoblast cell lines. Restored expression of let-7i inhibited the malignant phenotype of OS cells in vitro. Furthermore, it was shown that Aurora-B is a direct target of let-7i and it mediated the suppression of Aurora-B by binding to its 3'-UTR. Therefore, the present findings highlight the significance of let-7i as a tumor suppressor in cell malignant phenotype by targeting Aurora-B in OS.

The hsa-let-7i gene is a novel member of the let-7 miRNA family, and is located at $12 \mathrm{q} 14.1$. Although little is known 
regarding its function, recent studies have indicated that let-7i is a novel biomarker and therapeutic target in human epithelial ovarian cancer (16). Balakathiresan et al (17) revealed that its expression is elevated in the serum and cerebrospinal fluid of individuals who exposure to blast wave, suggesting it involve in blast-induced traumatic brain injury. However, let-7i expression is decreased in several types of malignancies $(8,11)$. Previous studies have shown that let-7 $\mathrm{i}$ is downregulated in ovarian cancer and may be used as a therapeutic target to modulate platinum-based chemotherapy and as a biomarker to predict chemotherapy response and survival $(16,18)$. Lai et al $(19)$ showed that aberrant expression of let-7i in $\mathrm{T}$ cells contributes to immunopathogenesis. Recently, a study showed that repression of bone morphogenetic protein 4 by let- $7 \mathrm{i}$ attenuates mesenchymal migration of head and neck cancer cells (11). Notably, Zhang et al (20) showed that mature hsa-let-7i expression was elevated and correlated with colorectal cancer metastasis. In the present study, it was demonstrated that let-7i was aberrantly expressed in OS tissues with pulmonary metastatic disease and cell lines, and restoration of let-7i inhibits the OS cell malignant phenotype in vitro.

Aurora-B is located on chromosome 17p13.1, a region that is not typically amplified in human malignancies. Increasing evidence shows that Aurora B is hypothesized to be an important antitumor target (21). Recently, studies have revealed that nuclear Aurora-B expression is strongly associated with tumor metastasis (22-25). Our previous study demonstrated that inhibition of Aurora-B suppress cell migration and invasion in OS cells (13). A recent study implicated Aurora-B as a target of let-7a, which contributes to the growth of endometrial carcinoma cells (14). Our results indicated that Aurora-B is the direct target of let-7i, which inhibited invasion and metastasis by binding the 3'-UTR of Aurora-B.

In conclusion, the present study demonstrated that let-7i can inhibit the malignant phenotype of OS cells by directly binding to the 3'-UTR of Aurora-B in vitro, suggesting that let-7i may be a novel potential target for OS treatment. However, tumor microenvironment may also be important in tumor development and metastasis. Thus, further experiments in vivo are required to be performed to investigate whether let-7i could act as a tumor inhibitor in OS.

\section{Acknowledgements}

The present study was supported by grants from the National Natural Science Foundation of China (grant no. 81360399), the Natural Science Foundation of Jiangxi Province (grant no. 2012ZBAB205016) and Jiangxi Province Education Department of Science and Technology (grant no. GJJ12097).

\section{References}

1. Hegyi M, Semsei AF, Jakab Z, et al: Good prognosis of localized osteosarcoma in young patients treated with limb-salvage surgery and chemotherapy. Pediatr Blood Cancer 57: 415-422, 2011.

2. Mialou V, Philip T, Kalifa C, et al: Metastatic osteosarcoma at diagnosis: prognostic factors and long-term outcome-the French pediatric experience. Cancer 104: 1100-1109, 2005.

3. Stokkel MP, Linthorst MF, Borm JJ, Taminiau AH and Pauwels EK: A reassessment of bone scintigraphy and commonly tested pretreatment biochemical parameters in newly diagnosed osteosarcoma. J Cancer Res Clin Oncol 128: 393-399, 2002.
4. Zhao H, Guo M, Zhao G, Ma Q, Ma B, Qiu X, et al: miR-183 inhibits the metastasis of osteosarcoma via downregulation of the expression of Ezrin in F5M2 cells. Int J Mol Med 30: 1013-1020, 2012

5. Wu X,Zhong D, Gao Q, Zhai W, Ding Z and Wu J: MicroRNA-34a inhibits human osteosarcoma proliferation by downregulating ether a go-go 1 expression. Int J Med Sci 10: 676-682, 2013.

6. Dong Q, Meng P, Wang T, Qin W, Wang F, Yuan J, et al: MicroRNA let-7a inhibits proliferation of human prostate cancer cells in vitro and in vivo by targeting E2F2 and CCND2. PLoS One 5: e10147, 2010.

7. Johnson SM, Grosshans H, Shingara J, Byrom M, Jarvis R, Cheng A, et al: RAS is regulated by the let-7 microRNA family. Cell 120: 635-647, 2005.

8. Takamizawa J, Konishi H, Yanagisawa K, Tomida S, Osada H, Endoh H, et al: Reduced expression of the let-7 microRNAs in human lung cancers in association with shortened postoperative survival. Cancer Res 64: 3753-3756, 2004.

9. Nadiminty N, Tummala R, Lou W, Zhu Y, Shi XB, Zou JX, et al: MicroRNA let-7c is downregulated in prostate cancer and suppresses prostate cancer growth. PLoS One 7: e32832, 2012.

10. De Vito C, Riggi N, Suva ML, Janiszewska M, Horlbeck J, Baumer K, et al: Let-7a is a direct EWS-FLI-1 target implicated in Ewing's sarcoma development. PLoS One 6: e23592, 2011.

11. Yang WH, Lan HY, Tai SK and Yang MH: Repression of bone morphogenetic protein 4 by let- $7 \mathrm{i}$ attenuates mesenchymal migration of head and neck cancer cells. Biochem Biophys Res Commun 433: 24-30, 2013.

12. Kelly AD, Haibe-Kains B, Janeway KA, Hill KE, Howe E, Goldsmith J, et al: MicroRNA paraffin-based studies in osteosarcoma reveal reproducible independent prognostic profiles at 14q32. Genome Med 5: 2, 2013.

13. Zhu XP, Liu ZL, Peng AF, Zhou YF, Long XH, Luo QF, et al: Inhibition of Aurora-B suppresses osteosarcoma cell migration and invasion. Exp Ther Med 7: 560-564, 2014.

14. Liu P, Qi M, Ma C, Lao G and Liu Y: Let7a inhibits the growth of endometrial carcinoma cells by targeting Aurora-B. FEBS Lett 587: 2523-2529, 2013.

15. Xie D, Shang C, Zhang H, Guo Y and Tong X: Up-regulation of miR-9 target CBX7 to regulate invasion ability of bladder transitional cell carcinoma. Med Sci Monit 21: 225-230, 2015.

16. Yang N, Kaur S, Volinia S, Greshock J, Lassus H, Hasegawa K, et al: MicroRNA microarray identifies Let-7i as a novel biomarker and therapeutic target in human epithelial ovarian cancer. Cancer Res 68: 10307-10314, 2008

17. Balakathiresan N, Bhomia M, Chandran R, Chavko M, McCarron RM and Maheshwari RK: MicroRNA let-7i is a promising serum biomarker for blast-induced traumatic brain injury. J Neurotrauma 29: 1379-1387, 2012.

18. Liu K, Qian T, Tang L, Wang J, Yang H and Ren J: Decreased expression of microRNA let-7i and its association with chemotherapeutic response in human gastric cancer. World J Surg Oncol 10: 225, 2012.

19. Lai NS, Yu HC, Chen HC, Yu CL, Huang HB and Lu MC: Aberrant expression of microRNAs in T cells from patients with ankylosing spondylitis contributes to the immunopathogenesis. Clin Exp Immunol 173: 47-57, 2013

20. Zhang P, Ma Y, Wang F, Yang J,Liu Z, Peng J, et al: Comprehensive gene and microRNA expression profiling reveals the crucial role of hsa-let-7i and its target genes in colorectal cancer metastasis. Mol Biol Rep 39: 1471-1478, 2012.

21. Zhang L and Zhang S: ZM447439, the Aurora kinase B inhibitor, suppresses the growth of cervical cancer SiHa cells and enhances the chemosensitivity to cisplatin. J Obstet Gynaecol Res 37: 591-600, 2011.

22. Takeshita M, Koga T, Takayama K, Ijichi K, Yano T, Maehara Y, et al: Aurora-B overexpression is correlated with aneuploidy and poor prognosis in non-small cell lung cancer. Lung Cancer 80: 85-90, 2013.

23. Tuncel H, Shimamoto F, Kaneko Guangying Qi H, Aoki E, Jikihara H, Nakai S, et al: Nuclear Aurora B and cytoplasmic Survivin expression is involved in lymph node metastasis of colorectal cancer. Oncol Lett 3: 1109-1114, 2012.

24. Pohl A, Azuma M, Zhang W, Yang D, Ning Y, Winder T, et al: Pharmacogenetic profiling of Aurora kinase B is associated with overall survival in metastatic colorectal cancer. Pharmacogenomics J 11: 93-99, 2011.

25. Qi G, Ogawa I, Kudo Y, Miyauchi M, Siriwardena BS, Shimamoto F, et al: Aurora-B expression and its correlation with cell proliferation and metastasis in oral cancer. Virchows Arch 450: 297-302, 2007. 